\title{
Cytotoxic Triterpenoids from the Stembark of Aglaia argentea (Meliaceae)
}

\author{
Ace Tatang Hidayat ${ }^{1,2}$, Kindi Farabi ${ }^{1}$, Desi Harneti ${ }^{1}$, Nurlelasari ${ }^{1}$, Rani Maharani ${ }^{1,2}$, \\ Ida Nurfarida ${ }^{2}$, Unang Supratman ${ }^{1,2,}$, and Yoshihito Shiono ${ }^{3}$ \\ ${ }^{1}$ Department of Chemistry, Faculty of Mathematics and Natural Sciences, Universitas Padjadjaran, \\ Jatinangor 45363, Indonesia \\ ${ }^{2}$ Central Laboratory of Universitas Padjadjaran, Jatinangor 45363, Indonesia \\ ${ }^{3}$ Department of Food, Life, and Environmental Science, Faculty of Agriculture, Yamagata University, \\ Tsuruoka, Yamagata 997-8555, Japan
}

Received May 5, 2017; Accepted November 18, 2017

\begin{abstract}
Four dammarane-type triterpenoid compounds, dammar-24-en-3a-ol (1), 3-epi-cabraleahydroxy lactone (2),

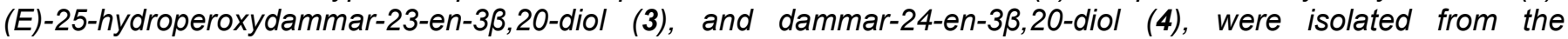
methanolic extract of the stembark of Aglaia argentea. Compounds, 1-4, were isolated for first time from this plant. The structure of isolated compounds were elucidated by spectroscopic methods including one and two-dimensional NMR as well as mass spectrometric analysis. Compounds 1-4, along with a known synthetic analog, 20-hydroxydammar-24-en-3-on (5), were evaluated their cytotoxic activity against $P$-388 murine leukemia cells in vitro. The $I C_{50}$ values of compounds, 1-5 were $9.09 \pm 0.10,68.53 \pm 0.08,5.89 \pm 0.08,22.40 \pm 0.11$, and $11.53 \pm 0.08 \mu \mathrm{g} / \mathrm{mL}$, respectively. Among the dammarane-type triterpenoids, compounds 1, 3, 4 and 5 having opened side chain showed the stronger activity, where's compound $\mathbf{2}$ with a cyclic side chain showed weak or no activity. In addition, compound 3 showed the strongest activity, indicate that hydroperoxy group at side chain increase cytotoxic activity.
\end{abstract}

Keywords: Aglaia argentea; cytotoxic activity; dammarane-type triterpenoids; P-388 murine leukemia

\section{ABSTRAK}

Empat senyawa triterpenoid tipe damaran, damar-24-en-3a-ol (1), 3-epi-kabraleahidroksi lakton (2), (E)-25-

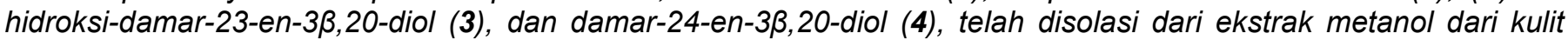
batang Aglaia argentea. Senyawa 1-4, diisolasi untuk pertama kali dari tumbuhan ini. Senyawa 1-4, bersama dengan senyawa analog sintetik yang telah dikenal, dammar-24-en,20-ol-3-on (5), dievaluasi aktivitas sitotoksiknya terhadap sel murin leukemia $P-388$ secara in vitro. Nilai $I C_{50}$ senyawa 1-5 berturut-turut adalah $9,09 \pm 0,10,68,53 \pm$ $0,08,5,89 \pm 0,08,22,40 \pm 0,11$, dan 11,53 $\pm 0,08 \mu \mathrm{g} / \mathrm{mL}$. Diantara senyawa triterpenoid tipe damaran, senyawa 1, 3, 4, dan 5 yang memiliki rantai samping terbuka menunjukkan aktivitas lebih kuat, sementara senyawa 2 dengan rantai samping siklik menunjukkan aktivitas lemah atau tidak aktif. Sebagai tambahan, senyawa $\mathbf{3}$ menunjukkan aktivitas sitotoksik paling kuat, mengindikasikan bahwa gugus hidroperoksi pada rantai samping meningkatkan aktivitas sitotoksik.

Kata Kunci: Aglaia argentea; aktivitas sitotoksik; triterpenoid tipe damaran; sel murin leukemia P-388

\section{INTRODUCTION}

Dammarane-type triterpenoids have been gaining worldwide attention for a long time because of their potent bioactivities [1]. Dammarane-type triterpenoids are distributed in genera of Panax (Araliaceae), Gynostemma (Cucurbitaceae), Aralia (Araliaceae), Aglaia (Meliaceae), Bacopa (Scrophulariaceae), Copernicia (Arecaceae), Celastrus (Celastraceae), Forsythia (Oleaceae), Myrica (Myrica), Rhus (Anacardiaceae), Polyscias (Araliaceae) and Sapindus (Sapindus) [2-6]. The basic molecular skeleton of dammarane-type triterpenoids comprises tetracyclic moiety and side chain moiety [1]. The bioactivities and chemical properties of dammarane-type triterpenoids have been investigated, and several pharmacological effects have been disclosed, including anti-fatigue, antihyperglycemic, antiobesity, anticancer, anti-HIV, antioxidant, antiaging, immunostimulatory, antiatherosclerotic and antihypertensive effects [7-9]. The genus Aglaia is the largest genus of the family of Meliaceae comprises more than 100 species distributed mainly in India, Indonesia, Malaysia and parts of the Western Pacific region [10]. The genus of

* Corresponding author.

DOI: 10.22146/ijc. 25052

Email address : unang.supratman@unpad.ac.id

Ace Tatang Hidayat et al. 
Aglaia traditionally used to moisturizing the lungs, reducing fever and for treating the contused wound, coughs and skin diseases [11]. In Indonesia, flowers of A. odorata traditionally used as an insect repellent [12].

Some species of Aglaia have been phytochemically investigated previously with unique biological activity, such as antifungal sesquiterpenoids [13-14], diterpenoids [15-16], insecticidal and cytotoxic rocaglate derivatives [17-18], lignans [19-20], cytotoxic and antiviral dammarane-type triterpenoids [21,24] and antitumor cycloartane-type triterpenoids [4-5,8].

In our continuous search for cytotoxic constituents against P-388 murine leukemia cells from Indonesian Aglaia plants, we isolated and described two new cytotoxic triterpenoids, aglinone, and aglinin $E$, from the bark of $A$. smithii [23], a new stigmastane steroid, 3,4epoxy-(22R,25)-tetrahydrofuran-stigmast-5-en, two new bisamide compounds, eximiamide $A$ and $B$, from the bark of $A$. eximia [24-25] and one lignan [20].

In the further screening for cytotoxic compounds from Indonesia Aglaia plants, we found that the $n$ hexane and ethyl acetate extract of $A$. argentea exhibited a cytotoxic activity against $\mathrm{P}$-388 murine leukemia cells with $\mathrm{IC}_{50}$ values of 26.72 and 15.49 $\mu \mathrm{g} / \mathrm{mL}$, respectively. We report herein the isolation, structural elucidation of triterpenoid compounds (1-4) and a synthetic analogue, $\mathbf{5}$, together with their cytotoxic activity.

\section{EXPERIMENTAL SECTION}

\section{Materials}

The bark of $A$. argentea was collected in Bogor Botanical Garden, Bogor, West Java Province, Indonesia in June 2015. The plant was identified by the staff of the Bogoriense Herbarium, Bogor, Indonesia and a voucher specimen (No. Bo-1288718) was deposited at the herbarium.

\section{Instrumentation}

Melting points were measured on an electrothermal melting point apparatus and are uncorrected. The IR spectra were recorded on a Perkin-Elmer Spectrum-100 FT-IR in $\mathrm{KBr}$. Mass spectra were obtained with a Synapt G2 mass spectrometer instrument. NMR data were recorded on a JEOL ECZ-600 spectrometer at $600 \mathrm{MHz}$ for ${ }^{1} \mathrm{H}$ and $150 \mathrm{MHz}$ for ${ }^{13} \mathrm{C}$ and $\mathrm{TMS}$ as an internal standard. Column chromatography was conducted on silica gel 60 (Kanto Chemical Co., Inc., Japan). TLC plates were precoated with silica gel GF 254 (Merck, 0.25 $\mathrm{mm}$ ) and detection was achieved by spraying with $10 \%$ $\mathrm{H}_{2} \mathrm{SO}_{4}$ in $\mathrm{EtOH}$, followed by heating.

\section{Procedure}

\section{Extraction and isolation}

The dried bark $(2.5 \mathrm{~kg})$ of $A$. argentea was extracted with methanol exhaustively $(12 \mathrm{~L})$ at room temperature for 5 days. After removal of the solvent under vacuum, the viscous concentrated of $\mathrm{MeOH}$ extract $(133.5 \mathrm{~g})$ was first suspended in $\mathrm{H}_{2} \mathrm{O}$ and then partitioned with $n$-hexane, EtOAc, and $n$-butanol, successively. The $n$-hexane soluble fraction $(26.3 \mathrm{~g})$ was fractionated by vacuum liquid chromatography on silica gel 60 using a gradient $n$-hexane and EtOAc to give nine fractions $(A-I)$. Fraction $C(2.68 \mathrm{~g})$ was chromatographed on a column of silica gel, eluted with a gradient of $n$-hexane-EtOAc (10:0-1:1), to give nine subfractions (C01-C09). Subfraction C05 was chromatographed on a column of silica gel, eluted with $\mathrm{CH}_{2} \mathrm{Cl}_{2}: \mathrm{CHCl}_{3}$ (9.75:0.25), to give four subfractions (C05A-C05D). Subfraction C05B was separated on preparative TLC, eluted with $n$-hexane-EtOAc (9:1), to give compound 1 (5.3 mg). Fraction D (1.65 g) was chromatographed on a column of silica gel, eluted with a gradient of $n$-hexane-EtOAc (10:1-1:10), to give six subfractions (D01-D06). Subfraction D04 was recrystallized in EtOAc, to give compound 2 (27.6 $\mathrm{mg}$ ). Subfraction D05 was chromatographed on a column of silica gel, eluted with a gradient of $n$-hexane-EtOAc $(10: 1-1: 10)$ to afford five subfractions (D05A-D05E). Subfraction D05D was chromatographed on a column of silica gel, eluted with a gradient of $\mathrm{CHCl}_{3}-\mathrm{EtOAC}$ $(10: 1-1: 10)$ to give compound $3(4.9 \mathrm{mg})$. The EtOAc soluble fraction $(12.1 \mathrm{~g})$ was fractionated by column chromatography on silica gel using a gradient $n$ hexane-EtOAc to give eight fractions (J-Q). Fraction $\mathrm{K}$ (927.6 mg) was chromatographed on a column of silica gel, eluted with $n$-hexane-EtOAc (10:1-1:1), to give four subfractions (K01-K04). Subfraction K02 was separated on preparative TLC silica gel $\mathrm{GF}_{254}$, eluted with $n$-hexane:EtOAc (7:3), to give compound 4 (40.3 $\mathrm{mg}$ )

Compound 4 (12.0 $\mathrm{mg})$ was dissolved in anhydrous pyridine $(1 \mathrm{~mL})$ in a vial $(4 \mathrm{~mL})$, and $\mathrm{CrO}_{3}$ $(20.0 \mathrm{mg}$ ) was then added. After standing at room temperature overnight, the reaction mixture was separated through a small silica gel $(1 \mathrm{~g})$ column $(0.5 \mathrm{x}$ $4.2 \mathrm{~cm})$, eluted with $n$-hexane:Me $2 \mathrm{CO}(4: 1,20 \mathrm{~mL})$. The elution was evaporated to dryness under reduced pressure at $45{ }^{\circ} \mathrm{C}$, to give the oxidation product of compound 5, 20-hydroxy-dammar-24-en-3-on (Rf 0.55; $4.2 \mathrm{mg}$ ).

Dammar-24-en-3 $\alpha$-ol (1). White needle-like crystals, m.p. $152-153{ }^{\circ} \mathrm{C}$; IR (KBr) $v_{\max } 3345,2937,2870,1464$, 1379, $1056 \mathrm{~cm}^{-1} ;{ }^{1} \mathrm{H}-\mathrm{NMR}\left(\mathrm{CDCl}_{3}, 600 \mathrm{MHz}\right)$ and ${ }^{13} \mathrm{C}-$ $\operatorname{NMR}\left(\mathrm{CDCl}_{3}, 125 \mathrm{MHz}\right)$, see Table 1. 


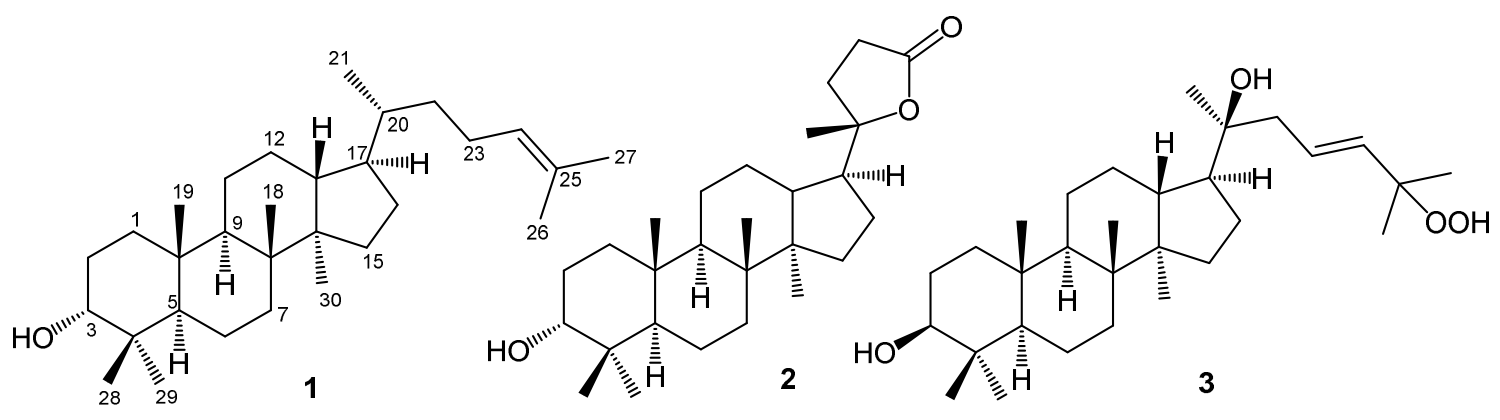

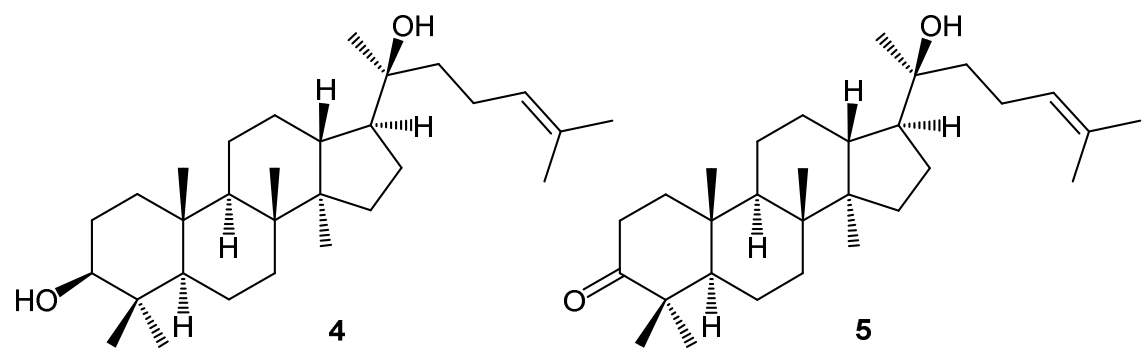

Fig 1. Structures of compounds 1-5

3-epi-Cabraleahydroxy lactone (2). White amorphous powder, m.p. (decomposed); IR (KBr) v $\max 3477,2942$, 1715, 1471, 1387, $1075 \mathrm{~cm}^{-1}$; ${ }^{1} \mathrm{H}-\mathrm{NMR}\left(\mathrm{CDCl}_{3}, 600\right.$ $\mathrm{MHz}),{ }^{13} \mathrm{C}-\mathrm{NMR}\left(\mathrm{CDCl}_{3}, 125 \mathrm{MHz}\right)$, see Table 1; HRTOFMS (positive ion mode) $\mathrm{m} / \mathrm{z} 417.3105[\mathrm{M}+\mathrm{H}]^{+}$, (calcd. $\mathrm{C}_{27} \mathrm{H}_{44} \mathrm{O}_{3}, m / z$ 416.3290).

(E)-25-Hydroperoxydammar-23-en-3 $\beta, 20$-diol

(3). Colorless oil; IR (KBr) $v_{\max } 3436,2945,1651,1456$, 1074, $847 \mathrm{~cm}^{-1}$; ${ }^{1} \mathrm{H}-\mathrm{NMR}\left(\mathrm{CDCl}_{3}, 600 \mathrm{MHz}\right),{ }^{13} \mathrm{C}-\mathrm{NMR}$ $\left(\mathrm{CDCl}_{3}, 125 \mathrm{MHz}\right.$ ), see Table 1; HR-TOFMS (positive ion mode) $m / z$ 477.3951 $[\mathrm{M}+\mathrm{H}]^{+}$, (calcd. $\mathrm{C}_{30} \mathrm{H}_{52} \mathrm{O}_{4}, \mathrm{~m} / \mathrm{z}$ 476.3866).

Dammar-24-en-3ß,20-diol (4). White amorphous powder, m.p. (decomposed); IR (KBr) vmax 3369, 2939, 1639, 1458, $1109 \mathrm{~cm}^{-1} ;{ }^{1} \mathrm{H}-\mathrm{NMR}\left(\mathrm{CDCl}_{3}, 600 \mathrm{MHz}\right),{ }^{13} \mathrm{C}-$ NMR $\left(\mathrm{CDCl}_{3}, 125 \mathrm{MHz}\right)$, see Table 2; HR-TOFMS (positive ion mode) $\mathrm{m} / \mathrm{z} 445.0527[\mathrm{M}+\mathrm{H}]^{+}$, (calcd. $\mathrm{C}_{30} \mathrm{H}_{52} \mathrm{O}_{2}, \mathrm{~m} / \mathrm{z}$ 444.3967).

20-Hydroxy-dammar-24-en-3-on (5). White amorphous powder, m.p. (decomposed); ${ }^{1} \mathrm{H}-\mathrm{NMR}\left(\mathrm{CDCl}_{3}, 600 \mathrm{MHz}\right)$ and ${ }^{13} \mathrm{C}-\mathrm{NMR}\left(\mathrm{CDCl}_{3}, 125 \mathrm{MHz}\right)$, see Table 2.

\section{Determination of Cytotoxic Activity}

The P388 murine leukemia cells were seeded into 96-well plates at an initial cell density of approximately $3 \times 10^{4}$ cells $\mathrm{cm}^{-3}$. After $24 \mathrm{~h}$ of incubation for cell attachment and growth, varying concentrations of samples were added. The compounds added were first dissolved in DMSO at the required concentration. Subsequent six desirable concentrations were prepared using PBS (phosphoric buffer solution, $\mathrm{pH}=7.30-7.65$ ). Control wells received only DMSO. The assay was terminated after a $48 \mathrm{~h}$ incubation period by adding MTT reagent [3-(4,5-dimethylthiazol-2-yl)-2,5-diphenyl tetra zolium bromide; also named as thiazol blue] and the incubation was continued for another $4 \mathrm{~h}$, in which the MTT-stop solution containing SDS (sodium dodecyl sulphate) was added and another $24 \mathrm{~h}$ incubation was conducted. Optical density was read by using a microplate reader at $550 \mathrm{~nm}$. $\mathrm{IC}_{50}$ values were taken from the plotted graph of percentage live cells compared to control (\%), receiving only PBS and DMSO, versus the tested concentration of compounds $(\mu \mathrm{g} / \mathrm{mL})$. The $\mathrm{IC}_{50}$ value is the concentration required for $50 \%$ growth inhibition. Each assay and analysis was run in triplicate and averaged [26-28].

\section{RESULT AND DISCUSSION}

Compound 1 was obtained as white needle-like crystal. The molecular formula of compound 1 was $\mathrm{C}_{30} \mathrm{H}_{52} \mathrm{O}$ based on the analysis of NMR and thus required five degrees of unsaturation, originating from one pair of $\mathrm{C} s p^{2}$ and the remaining tetracyclic triterpenoids. The IR spectra showed absorption peaks at $3345 \mathrm{~cm}^{-1}(\mathrm{OH}), 2937$ and $\left.2870 \mathrm{~cm}^{-1}(\mathrm{C}-\mathrm{H} \mathrm{sp})^{3}\right)$, $1464 \mathrm{~cm}^{-1}(\mathrm{C}=\mathrm{C}), 1379 \mathrm{~cm}^{-1}$ (gem-dimethyl groups), and $1056 \mathrm{~cm}^{-1}$ (C-O). The ${ }^{1} \mathrm{H}-\mathrm{NMR}\left(\mathrm{CDCl}_{3} 600 \mathrm{MHz}\right)$ spectrum showed the presence of seven tertiary methyl groups, resonating at $\delta_{\mathrm{H}} 0.95(\mathrm{H}-18), 0.85(\mathrm{H}-19)$, $1.62(\mathrm{H}-26), 1.56(\mathrm{H}-27), 0.96(\mathrm{H}-28), 0.79(\mathrm{H}-29)$, and $0.88(\mathrm{H}-30)$ and one secondary methyl at $\delta_{H} 1.10(\mathrm{~d}$, $J=6.5 \mathrm{~Hz}, \mathrm{H}-21)$. There was one olefinic methine group, resonating at $\delta_{\mathrm{H}} 5.09(1 \mathrm{H}, \mathrm{t}, \mathrm{J}=7 \mathrm{~Hz}, \mathrm{H}-24)$ and 

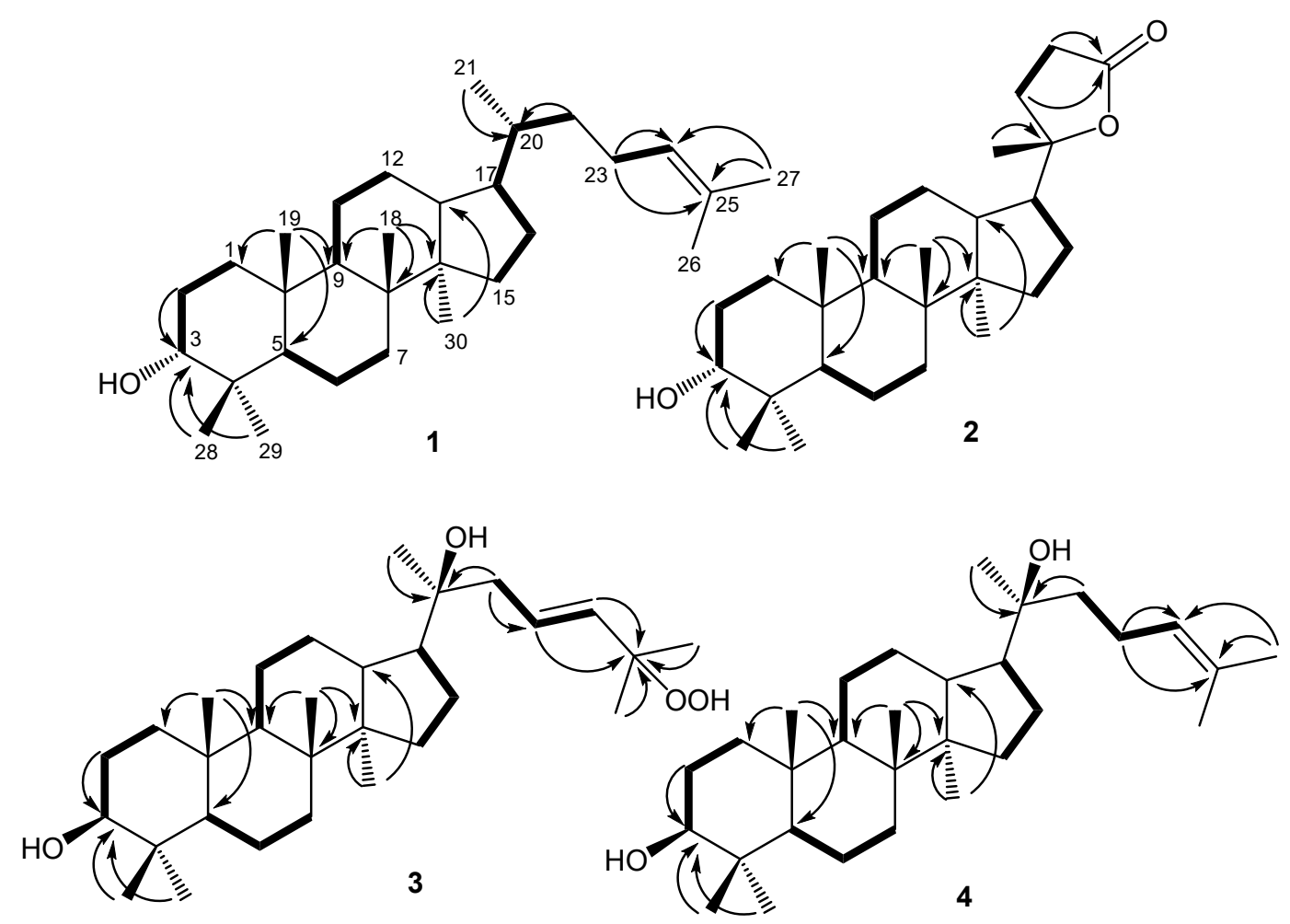

Fig 2. Selected $\mathrm{HMBC}$ and ${ }^{1} \mathrm{H}-{ }^{1} \mathrm{H}$ COSY correlations for $1-4$

one oxymethine resonating at $\delta_{H} 3.64(1 \mathrm{H}, \mathrm{d}, J=2.5 \mathrm{~Hz}$, $\mathrm{H}-3)$ which indicates that the hydroxy group was attached in $\mathrm{C}-3$. The proton pairing was also confirmed with the ${ }^{1} \mathrm{H}-{ }^{1} \mathrm{H}$ COSY spectrum (Fig. 2). The ${ }^{13} \mathrm{C}-\mathrm{NMR}$ $\left(\mathrm{CDCl}_{3} 150 \mathrm{MHz}\right)$ and DEPT $135^{\circ}$ spectra showed the presence of eight methyl groups, exhibiting the characteristics of triterpenoid compounds [21-22], one olefinic methine at $\delta c 125.3$ (C-24), one olefinic quaternary carbon at $\delta_{c} 130.5$ (C-25), and an oxymethine group at $\delta c 75.0$ (C-3). The HMBC crosspeaks (Fig. 2) from $\mathrm{CH}_{3}-28$ ( $\left.\delta_{\mathrm{H}} 0.96\right), \mathrm{CH}_{3}-29$ (ठн 0.79$)$, and the methylene protons at $\mathrm{H}-2\left(\delta_{\mathrm{H}} 1.47\right)$ to the oxymethine carbon at $\mathrm{C}-3$ ( $\delta_{c}$ 75.0) indicated the presence of a hydroxy group at $\mathrm{C}-3$. The correlation which was arising from $\mathrm{CH}_{3}-26\left(\delta_{\mathrm{H}} 1.62\right)$ and $\mathrm{CH}_{3}-27\left(\delta_{\mathrm{H}}\right.$ $1.56)$ to $C-25$ ( $\left.\delta_{c} 130.5\right)$ and $C-24\left(\delta_{c} 124.3\right)$ indicated that position of a double bond at C-24/C-25. The conformation of $\mathrm{C}-3$ was assigned as a based on coupling constant of $\mathrm{H}-3(\mathrm{~J}=2.6 \mathrm{~Hz})$ and by comparing to those of reference [29]. These functionalities accounted for one of five total degrees of unsaturation, and the remaining four degrees of unsaturation were consistent with the triterpenoid skeleton. A comparison of the NMR data of 1 with dammar-24-en-3a-ol [30] revealed that the structures of the two compounds were very similar; consequently, compound 1 was identified as dammar-24-en-3a-ol.
Compound 2 was obtained as a white amorphous powder. Its molecular composition $\mathrm{C}_{27} \mathrm{H}_{44} \mathrm{O}_{3}$, was established from the HR-ESI-TOFMS spectrum $(\mathrm{m} / \mathrm{z}$ 417.3105, $[\mathrm{M}+\mathrm{H}]^{+}$) together with NMR data (Table 1). The IR spectra showed absorption peaks at $3477 \mathrm{~cm}^{-1}$ $\left.(\mathrm{OH}), 2942 \mathrm{~cm}^{-1}(\mathrm{C}-\mathrm{H} \mathrm{sp})^{3}\right), 1715 \mathrm{~cm}^{-1}(\mathrm{C}=\mathrm{O}), 1471$ and $1379 \mathrm{~cm}^{-1}$ (gem-dimethyl groups), and $1075 \mathrm{~cm}^{-1}$ (C-O). The ${ }^{1} \mathrm{H}-\mathrm{NMR}\left(\mathrm{CDCl}_{3} 600 \mathrm{MHz}\right)$ spectrum showed the presence of six tertiary methyl groups, resonating at $\delta_{\mathrm{H}} 0.92\left(\mathrm{CH}_{3}-18\right), 0.82\left(\mathrm{CH}_{3}-19\right)$, $1.33\left(\mathrm{CH}_{3}-21\right), 0.91\left(\mathrm{CH}_{3}-28\right), 0.81\left(\mathrm{CH}_{3}-29\right)$, and $0.87\left(\mathrm{CH}_{3}-30\right)$ and one oxymethine group, resonating at $\delta_{\mathrm{H}} 3.37(1 \mathrm{H}, \mathrm{s}, \mathrm{H}-3)$ which was indicated the presence of dammarane-type triterpenoid skeleton [30-31]. The proton pairing was also confirmed with the ${ }^{1} \mathrm{H}-{ }^{1} \mathrm{H}$ COSY spectrum (Fig. 2). The ${ }^{13} \mathrm{C}-\mathrm{NMR}\left(\mathrm{CDCl}_{3} 150 \mathrm{MHz}\right)$ spectra showed 27 carbons and classified by DEPT $135^{\circ}$ experiment as six methyl groups, exhibiting the characteristics of trisnor-triterpenoid compounds [31], one carbonyl lactone at $\delta_{c} 176.9$ (C-24), an oxymethine group at $\delta_{c} 75.0(\mathrm{C}-3)$, and an oxygenated quaternary carbon at $\delta_{c} 90.3$ (C-20). The HMBC cross-peaks (Fig. 2) from $\mathrm{CH}_{3}-28\left(\delta_{\mathrm{H}} 0.91\right), \mathrm{CH}_{3}-29\left(\delta_{\mathrm{H}} 0.81\right)$, and the methylene protons at $\mathrm{H}-2\left(\delta_{\mathrm{H}} 1.40\right)$ to the oxymethine carbon at C-3 ( $\delta_{c}$ 76.3) indicated the presence of a hydroxy group at $\mathrm{C}-3$. The correlation which was arising from $\mathrm{H}-22\left(\delta_{\mathrm{H}} 1.47\right)$ and $\mathrm{H}-23\left(\delta_{\mathrm{H}} 2.52\right)$ to $\mathrm{C}-24$ ( $\left.\delta_{c} 176.9\right)$ and C-20 ( $\delta_{c}$ 90.3) indicated that position of 
Table 1. NMR Data (600 MHz for ${ }^{1} \mathrm{H}$ and $150 \mathrm{MHz}$ for ${ }^{13} \mathrm{C}$, in $\mathrm{CDCl}_{3}$ ) for $1-5$

\begin{tabular}{|c|c|c|c|c|c|c|c|c|c|}
\hline \multirow[b]{2}{*}{ No. } & 1 & \multicolumn{2}{|c|}{2} & \multicolumn{2}{|c|}{3} & \multicolumn{2}{|r|}{4} & \multicolumn{2}{|r|}{5} \\
\hline & $\begin{array}{l}{ }^{13} \mathrm{C} \text { C-NMR } \\
\text { סc (mult.) }\end{array}$ & $\begin{array}{r}\text { 'H-NMR } \\
\delta_{H}(\text { Int., mult, } \\
J=H z) \\
\end{array}$ & 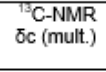 & $\begin{array}{r}{ }^{1} \mathrm{H}-\mathrm{NMMR} \\
\delta_{\mathrm{H}} \text { (Int), mult, } J=\mathrm{Hz} \text { ) }\end{array}$ & $\begin{array}{l}{ }^{73} \mathrm{C}-\mathrm{NMMR} \\
\bar{\delta} \mathrm{c} \text { (mult)) }\end{array}$ & $\begin{array}{r}\text { 'H-NMR } \\
\delta_{H} \text { (Int., mult, } J=\mathrm{Hz} \text { ) }\end{array}$ & $\begin{array}{l}{ }^{13} \mathrm{C}-\mathrm{NMMR} \\
{ }_{\delta} \mathrm{c} \text { (mult.) }\end{array}$ & $\begin{array}{r}\text { 'H-NMR } \\
\delta_{\mathrm{H}} \text { (Int., mult, } J=\mathrm{Hz} \text { ) }\end{array}$ & $\begin{array}{r}{ }^{1} \mathrm{H}-\mathrm{NMMR} \\
\delta_{\mathrm{H}} \text { (Int., mult, } J=\mathrm{Hz} \text { ) }\end{array}$ \\
\hline 1 & $33.7(t)$ & $1.33(2 \mathrm{H}, \mathrm{m})$ & $35.2(\mathrm{t})$ & $1.17,1.50($ each $1 \mathrm{H}, \mathrm{m})$ & $39.1(\mathrm{t})$ & $1.68(2 \mathrm{H}, \mathrm{dd}, 3.6,13.2)$ & $33.7(\mathrm{t})$ & $1.37,1.40($ each $1 \mathrm{H}, \mathrm{d}, 1.2)$ & $1.44,1.93($ each $1 \mathrm{H}, \mathrm{m})$ \\
\hline 2 & $24.6(t)$ & $1.47(2 \mathrm{H}, \mathrm{m})$ & $33.7(t)$ & $1.40(2 \mathrm{H}, \mathrm{dd}, 2.4,9.6)$ & $24.9(\mathrm{t})$ & $1.44,1.71($ each $1 \mathrm{H}, \mathrm{m})$ & $24.9(\mathrm{t})$ & $1.43(2 \mathrm{H}, \mathrm{m})$ & $1.96,2.48($ each $1 \mathrm{H}, \mathrm{m})$ \\
\hline 3 & 75.0 (d) & $3.64(1 \mathrm{H}, \mathrm{d}, 2.6)$ & 76.3 (d) & $3.37(1 \mathrm{H}, \mathrm{s})$ & 79.1 (d) & $3.19(1 \mathrm{H}, \mathrm{dd}, 4.8,11.4)$ & 76.4 (d) & $3.37(1 \mathrm{H}, \mathrm{t}, 4.5)$ & - \\
\hline 4 & $37.5(\mathrm{~s})$ & 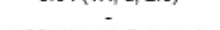 & $37.3(\mathrm{~s})$ & (n) & $39.0(\mathrm{~s})$ & & $37.7(\mathrm{~s})$ & & \\
\hline 5 & 49.2 (d) & $1.36(1 \mathrm{H}, \mathrm{dd}, 2.4,11.4)$ & 49.4 (d) & $1.95(1 \mathrm{H}, \mathrm{m})$ & 55.9 (d) & $0.71(1 \mathrm{H}, \mathrm{dd}, 2.4,9.6)$ & 49.6 (d) & $1.23(1 \mathrm{H}, \mathrm{m})$ & $1.39(1 \mathrm{H}, \mathrm{t}, 5.3)$ \\
\hline 6 & $18.1(t)$ & $1.40(2 \mathrm{H}, \mathrm{m})$ & $18.3(\mathrm{t})$ & $1.37(2 \mathrm{H}, \mathrm{m})$ & $18.3(\mathrm{t})$ & $1.40,1.53($ each $1 \mathrm{H}, \mathrm{m})$ & $18.3(\mathrm{t})$ & $1.38(2 \mathrm{H}, \mathrm{m})$ & $1.46(2 \mathrm{H}, \mathrm{m})$ \\
\hline 7 & $35.2(\mathrm{t})$ & $1.23(2 \mathrm{H}, \mathrm{m})$ & $26.9(\mathrm{t})$ & $1.71(2 \mathrm{H}, \mathrm{m})$ & $35.3(\mathrm{t})$ & $1.25(2 \mathrm{H}, \mathrm{m})$ & $35.2(\mathrm{t})$ & $1.24,1.55($ each $1 \mathrm{H}, \mathrm{m})$ & $1.33(2 \mathrm{H}, \mathrm{m})$ \\
\hline 8 & $40.6(\mathrm{~s})$ & & $40.6(\mathrm{~s})$ & - & $40.4(\mathrm{~s})$ & - & $40.7(\mathrm{~s})$ & . & - \\
\hline 9 & 49.5 (d) & $1.71(1 \mathrm{H}, \mathrm{t}, 4.8)$ & 50.4 (d) & $1.41(1 \mathrm{H}, \mathrm{dd}, 2.4,13.2)$ & 50.7 (d) & $1.29(1 \mathrm{H}, \mathrm{m})$ & 50.4 (d) & $1.42(1 \mathrm{H}, \mathrm{m})$ & $1.41(1 \mathrm{H}, \mathrm{t}, 5.3)$ \\
\hline 10 & 37.2 (d) & $-1+2$ & $37.7(\mathrm{~s})$ & - & $37.2(\mathrm{~s})$ & $\because$ & $37.3(\mathrm{~s})$ & - & - \\
\hline 11 & $21.3(\mathrm{t})$ & $1.51(2 \mathrm{H}, \mathrm{m})$ & $25.4(\mathrm{t})$ & $1.20(2 \mathrm{H}, \mathrm{m})$ & $21.6(\mathrm{t})$ & $1.22,1.48($ each $1 \mathrm{H}, \mathrm{m})$ & $21.4(\mathrm{t})$ & $1.52(2 \mathrm{H}, \mathrm{m})$ & $1.54(2 \mathrm{H}, \mathrm{m})$ \\
\hline 12 & $25.7(\mathrm{t})$ & $1.09(2 \mathrm{H}, \mathrm{m})$ & $21.3(t)$ & $1.49,1.91($ each $1 \mathrm{H}, \mathrm{m})$ & $27.5(t)$ & $1.59(2 \mathrm{H}, \mathrm{m})$ & $25.4(\mathrm{t})$ & $\begin{array}{c}1.53(1 \mathrm{H}, \mathrm{m}) \\
1.91(1 \mathrm{H}, \mathrm{tt}, 2.4,13.1)\end{array}$ & $1.91(2 \mathrm{H}, \mathrm{m})$ \\
\hline 13 & 42.0 (d) & $1.71(1 \mathrm{H}, \mathrm{m})$ & 43.2 (d) & $1.53(1 \mathrm{H}, \mathrm{m})$ & 42.5 (d) & $1.63(1 \mathrm{H}, \mathrm{m})$ & 42.3 (d) & $1.58(1 \mathrm{H}, \mathrm{m})$ & $1.69(1 \mathrm{H}, \mathrm{m})$ \\
\hline 14 & $50.3(\mathrm{~s})$ & - & $50.3(\mathrm{~s})$ & - & $50.4(\mathrm{~s})$ & - & $50.5(\mathrm{~s})$ & - & - \\
\hline 15 & $31.1(t)$ & $1.00(2 \mathrm{H}, \mathrm{m})$ & $31.2(t)$ & $1.90,1.10($ each $1 \mathrm{H}, \mathrm{m})$ & $31.2(t)$ & $\begin{array}{c}1.07(1 \mathrm{H}, \mathrm{dd}, 1.8,8.4) \\
1.45(1 \mathrm{H}, \mathrm{m})\end{array}$ & $31.2(t)$ & $\begin{array}{c}1.04(1 \mathrm{H}, \mathrm{dd}, 7.2,11.4) \\
1.45(1 \mathrm{H}, \mathrm{m})\end{array}$ & $1.61,2.01$ (each $1 \mathrm{H}, \mathrm{m}$ ) \\
\hline 16 & $27.7(\mathrm{t})$ & $1.13(2 \mathrm{H}, \mathrm{m})$ & $25.1(\mathrm{t})$ & $1.52(2 \mathrm{H}, \mathrm{m})$ & $27.6(t)$ & $1.21,1.82($ each $1 \mathrm{H}, \mathrm{m})$ & $27.6(\mathrm{t})$ & $1.77(2 \mathrm{H}, \mathrm{m})$ & $1.51,1.98($ each $1 \mathrm{H}, \mathrm{m})$ \\
\hline 17 & 50.6 (d) & $1.46(1 \mathrm{H}, \mathrm{m})$ & 49.5 (d) & $1.23(1 \mathrm{H}, \mathrm{m})$ & 50.3 (d) & $1.72(1 \mathrm{H}, \mathrm{dd}, 3.6,6.6)$ & 49.8 (d) & $1.69(1 \mathrm{H}, \mathrm{m})$ & $1.71(1 \mathrm{H}, \mathrm{m})$ \\
\hline 18 & $15.8(q)$ & $0.95(3 \mathrm{H}, \mathrm{s})$ & $15.6(q)$ & $0.92(3 \mathrm{H}, \mathrm{s})$ & $15.6(q)$ & $0.94(3 \mathrm{H}, \mathrm{s})$ & $15.6(q)$ & $0.93(3 \mathrm{H}, \mathrm{s})$ & $0.94(3 \mathrm{H}, \mathrm{s})$ \\
\hline 19 & $15.1(q)$ & $0.85(3 \mathrm{H}, \mathrm{s})$ & $16.1(q)$ & $0.82(3 \mathrm{H}, \mathrm{s})$ & 16.5 (q) & $0.83(3 \mathrm{H}, \mathrm{s})$ & $16.1(q)$ & $0.82(3 \mathrm{H}, \mathrm{s})$ & $0.83(3 \mathrm{H}, \mathrm{s})$ \\
\hline 20 & 38.7 (d) & $1.16(1 \mathrm{H}, \mathrm{m})$ & $90.3(\mathrm{~s})$ & - & $75.2(\mathrm{~s})$ & - & $75.5(\mathrm{~s})$ & - & - \\
\hline 21 & 25.1 (q) & $1.10(3 \mathrm{H}, \mathrm{d}, 6.5)$ & 25.4 (q) & $1.33(3 \mathrm{H}, \mathrm{s})$ & 25.8 (q) & $1.11(3 \mathrm{H}, \mathrm{s})$ & 25.5 (q) & $1.13(3 \mathrm{H}, \mathrm{s})$ & $1.15(3 \mathrm{H}, \mathrm{s})$ \\
\hline 22 & $41.2(\mathrm{t})$ & $1.42(2 \mathrm{H}, \mathrm{m})$ & $31.3(\mathrm{t})$ & $1.47,2.01($ each $1 \mathrm{H}, \mathrm{m})$ & $43.4(\mathrm{t})$ & $2.22(2 \mathrm{H}, \mathrm{dd}, 7.8,11.4)$ & $40.6(\mathrm{t})$ & $1.44(2 \mathrm{H}, \mathrm{m})$ & $1.45(2 \mathrm{H}, \mathrm{m})$ \\
\hline 23 & $22.5(t)$ & $1.24(2 \mathrm{H}, \mathrm{m})$ & $29.3(t)$ & $\begin{array}{l}2.52(1 \mathrm{H}, \mathrm{d}, 10) \\
2.62(1 \mathrm{H}, \mathrm{d}, 9.9)\end{array}$ & 127.4 (d) & $5.76(1 \mathrm{H}, \mathrm{dd}, 7.8,16.2)$ & $22.6(t)$ & $2.02(2 \mathrm{H}, \mathrm{q}, 7.8)$ & $2.01(2 \mathrm{H}, \mathrm{q}, 7.8)$ \\
\hline $\begin{array}{l}24 \\
25\end{array}$ & $\begin{array}{l}125.3(d) \\
130.5(s)\end{array}$ & $5.09(1 \mathrm{H}, \mathrm{t}, 7.0)$ & $176.9(\mathrm{~s})$ & ${ }^{3}$ & $\begin{array}{l}137.4 \text { (d) } \\
82.2(\mathrm{~s})\end{array}$ & $5.60(1 \mathrm{H}, \mathrm{dd}, 4.8,16.2)$ & $\begin{array}{l}124.8 \text { (d) } \\
131.7(\mathrm{~s})\end{array}$ & $5.10(1 \mathrm{H}, \mathrm{t}, 5.4)$ & $5.13(1 \mathrm{H}, \mathrm{t}, 5.4)$ \\
\hline 26 & $\begin{array}{l}130.5(\mathrm{~s}) \\
25.2(\mathrm{q})\end{array}$ & $1.62(3 \mathrm{H}, \mathrm{s})$ & & & $\begin{array}{l}82.2(\mathrm{~s}) \\
24.2(\mathrm{q})\end{array}$ & $1.34(3 \mathrm{H}, \mathrm{s})$ & $\begin{array}{l}131.7(\mathrm{~s}) \\
25.9(\mathrm{q})\end{array}$ & $1.66(3 \mathrm{H}, \mathrm{s})$ & $1.65(3 \mathrm{H}, \mathrm{s})$ \\
\hline 27 & $16.9(q)$ & $1.56(3 \mathrm{H}, \mathrm{s})$ & & & $24.5(q)$ & $1.33(3 \mathrm{H}, \mathrm{s})$ & $17.8(q)$ & $1.59(3 \mathrm{H}, \mathrm{s})$ & $1.50(3 \mathrm{H}, \mathrm{s})$ \\
\hline 28 & 28.3 (q) & $0.96(3 \mathrm{H}, \mathrm{s})$ & $28.4(q)$ & $0.91(3 \mathrm{H}, \mathrm{s})$ & 28.1 (q) & $0.96(3 \mathrm{H}, \mathrm{s})$ & 28.4 (q) & $0.91(3 \mathrm{H}, \mathrm{s})$ & $0.92(3 \mathrm{H}, \mathrm{s})$ \\
\hline 29 & $21.8(q)$ & $0.79(3 \mathrm{H}, \mathrm{s})$ & 22.2 (q) & $0.81(3 \mathrm{H}, \mathrm{s})$ & 15.4 (q) & $0.76(3 \mathrm{H}, \mathrm{s})$ & $22.2(q)$ & $0.81(3 \mathrm{H}, \mathrm{s})$ & $0.83(3 \mathrm{H}, \mathrm{s})$ \\
\hline 30 & $16.2(q)$ & $0.88(3 \mathrm{H}, \mathrm{s})$ & $16.4(\mathrm{q})$ & $0.87(3 \mathrm{H}, \mathrm{s})$ & 16.3 (q) & $0.85(3 \mathrm{H}, \mathrm{s})$ & $16.6(q)$ & $0.86(3 \mathrm{H}, \mathrm{s})$ & $0.86(3 \mathrm{H}, \mathrm{s})$ \\
\hline
\end{tabular}

lactone in C-20/C-24. The conformation of C-3 was assigned as $\alpha$ based on coupling constant of $\mathrm{H}-3(\mathrm{~J}=0)$ [22,31]. These functionalities accounted for one of six total degrees of unsaturation, and the remaining five degrees of unsaturation were consistent with the triterpenoid skeleton with lactone ring at the side chain. A comparison of the NMR data of $\mathbf{2}$ with cabraleahydroxy lactone [22] revealed that the structures of the two compounds were very similar, consequently, compound 2 was identified as a 3-epi-cabraleahydroxy lactone.

Compound 3 was obtained as a colorless oil. Its molecular composition $\mathrm{C}_{30} \mathrm{H}_{52} \mathrm{O}_{4}$, was established from the HR-ESI-TOFMS spectrum $\left(\mathrm{m} / \mathrm{z}\right.$ 477.3951, $\left.[\mathrm{M}+\mathrm{H}]^{+}\right)$ together with NMR data (Table 1). The IR spectra showed absorption peaks at $3436 \mathrm{~cm}^{-1}(\mathrm{OH}), 2945 \mathrm{~cm}^{-1}$ $\left(\mathrm{C}-\mathrm{H} \mathrm{sp}{ }^{3}\right), 1651 \mathrm{~cm}^{-1}(\mathrm{C}=\mathrm{C}), 1456 \mathrm{~cm}^{-1}$ (gem-dimethyl groups), $1076 \mathrm{~cm}^{-1}$ (C-O), and $847 \mathrm{~cm}^{-1}(\mathrm{O}-\mathrm{O})$. The ${ }^{1} \mathrm{H}-$ NMR $\left(\mathrm{CDCl}_{3} 600 \mathrm{MHz}\right)$ spectrum showed the presence of eight tertiary methyl groups, resonating at $\delta_{\mathrm{H}} 0.94$ $\left(\mathrm{CH}_{3}-18\right), 0.83\left(\mathrm{CH}_{3}-19\right), 1.11\left(\mathrm{CH}_{3}-21\right), 1.34\left(\mathrm{CH}_{3}-26\right)$, $1.33\left(\mathrm{CH}_{3}-27\right), 0.96\left(\mathrm{CH}_{3}-28\right), 0.76\left(\mathrm{CH}_{3}-29\right)$, and 0.85 $\left(\mathrm{CH}_{3}-30\right)$, one oxymethine group, resonating at $\delta_{\mathrm{H}} 3.19$ $(1 \mathrm{H}, \mathrm{dd}, J=4.8,11.4 \mathrm{~Hz}, \mathrm{H}-3)$, and two methine $s p^{2}$ at $\delta_{H} 5.76(1 \mathrm{H}, \mathrm{dd}, J=7.8,16.2 \mathrm{~Hz})$ and $5.60(1 \mathrm{H}, \mathrm{dd}, J=$ $4.8,16.2 \mathrm{~Hz}, \mathrm{H}-24)$, which was indicated the presence of dammarane-type triterpenoid skeleton. The proton pairing was also confirmed with the ${ }^{1} \mathrm{H}-{ }^{1} \mathrm{H}$ COSY spectrum (Fig. 2). The ${ }^{13} \mathrm{C}-\mathrm{NMR} \quad\left(\mathrm{CDCl}_{3} 150 \mathrm{MHz}\right)$ spectra showed 30 carbons and classified by DEPT $135^{\circ}$ experiment as eight methyl groups, an oxymethine group at $\delta c 79.1$ (C-3), two oxygenated quaternary carbons at $\delta_{c} 75.2(\mathrm{C}-20)$ and $82.2(\mathrm{C}-24)$, and two methine $s p^{2}$ at ठc 127.4 (C-23) and 137.4 (C-24). One oxygenated quaternary carbon at $\delta_{c} 82.2$ (C-24) was more deshielded, indicate that hydroperoxy group attaches at $\mathrm{C}-24$ [26]. The HMBC cross-peaks (Fig. 2) from $\mathrm{H}-28$ $\left(\delta_{H} 0.96\right), \mathrm{H}-29\left(\delta_{H} 0.76\right)$, and the methylene protons at $\mathrm{H}-2\left(\delta_{\mathrm{H}} 1.44\right)$ to the oxymethine carbon at C-3 ( $\left.\delta_{\mathrm{C}} 79.1\right)$ indicated the presence of a hydroxy group at C-3. The correlation which was arising from $\mathrm{H}-23\left(\delta_{\mathrm{H}} 5.76\right)$ and $\mathrm{H}-24\left(\delta_{H} 5.60\right)$ to $\mathrm{C}-22$ ( $\left.\delta_{c} 43.4\right)$ and $\mathrm{C}-25$ ( $\delta_{c}$ 82.2) suggest the position of a double bond at C-23/C-24. The conformation of $\mathrm{C}-3$ was assigned as $\beta$ based on coupling constant of $\mathrm{H}-3(J=4.8,11.4 \mathrm{~Hz})$ [22]. These functionalities accounted for one of five total degrees of unsaturation, and the remaining four degrees of unsaturation were consistent with the triterpenoid skeleton. A comparison of the NMR data of 3 with $(E)$ 25-hydroperoxydammar-23-en-3 $\beta, 20$-diol [22] revealed that the structures of the two compounds were very similar; consequently, compound $\mathbf{3}$ was identified as 3(E)-25-hydroperoxydammar-23-en-3 $\beta, 20$-diol.

Compound 4 was obtained as a colorless oil. Its molecular composition $\mathrm{C}_{30} \mathrm{H}_{52} \mathrm{O}_{2}$, was established from the HR-ESI-TOFMS spectrum $\left(\mathrm{m} / \mathrm{z}\right.$ 445.0527, $\left.[\mathrm{M}+\mathrm{H}]^{+}\right)$ together with NMR data (Table 1). The IR spectra showed absorption peaks at $3369 \mathrm{~cm}^{-1}(\mathrm{OH}), 2939 \mathrm{~cm}^{-1}$ (C-H sp $), 1639 \mathrm{~cm}^{-1}(\mathrm{C}=\mathrm{C}), 1458 \mathrm{~cm}^{-1}$ (gem-dimethyl groups), and $1109 \mathrm{~cm}^{-1}(\mathrm{C}-\mathrm{O})$. The ${ }^{1} \mathrm{H}-\mathrm{NMR}\left(\mathrm{CDCl}_{3}\right.$ $600 \mathrm{MHz}$ ) spectrum showed the presence of eight tertiary methyl groups, resonating at $\delta_{\mathrm{H}} 0.93\left(\mathrm{CH}_{3}-18\right)$, $0.82\left(\mathrm{CH}_{3}-19\right), 1.13\left(\mathrm{CH}_{3}-21\right), 1.66\left(\mathrm{CH}_{3}-26\right), 1.59\left(\mathrm{CH}_{3}-\right.$ $27), 0.91\left(\mathrm{CH}_{3}-28\right), 0.81\left(\mathrm{CH}_{3}-29\right)$, and $0.86\left(\mathrm{CH}_{3}-30\right)$, 
Table 2. Cytotoxic activity of compounds $\mathbf{1}-\mathbf{5}$ against P-388 murine leukemia cells

\begin{tabular}{|c|c|}
\hline Compounds & $\mathrm{IC}_{50}(\mu \mathrm{g} / \mathrm{mL})$ \\
\hline Dammar-24-en-3a-ol (1) & $9.09 \pm 0.10$ \\
\hline 3-epi-Cabraleahydroxy lactone (2) & $68.53 \pm 0.08$ \\
\hline (E)-25-Hydroperoxydammar-23-en-3 $\beta, 20$-diol (3) & $5.89 \pm 0.08$ \\
\hline Dammar-24-en-3 $\beta, 20$-diol (4) & $22.40 \pm 0.11$ \\
\hline 20-Hydroxy-dammar-24-en-3-on (5) & $11.53 \pm 0.08$ \\
\hline
\end{tabular}

one oxymethine group, resonating at $\delta_{\mathrm{H}} 3.37(1 \mathrm{H}, \mathrm{t}, \mathrm{J}=$ $4.5 \mathrm{~Hz}, \mathrm{H}-3)$, and one methine $s p^{2}$ at $\delta_{H} 5.10(1 \mathrm{H}, \mathrm{t}, \mathrm{J}=$ $5.4 \mathrm{~Hz}, \mathrm{H}-24)$ which was indicated the presence of dammarane-type triterpenoid skeleton. The proton pairing was also confirmed with the ${ }^{1} \mathrm{H}-{ }^{1} \mathrm{H}$ COSY spectrum (Fig. 2). The ${ }^{13} \mathrm{C}-\mathrm{NMR} \quad\left(\mathrm{CDCl}_{3}\right.$ $150 \mathrm{MHz}$ ) spectra showed 30 carbons and classified by DEPT $135^{\circ}$ experiment as eight methyl groups, an oxymethine group at $\delta_{c} 76.4$ (C-3), one oxygenated quaternary carbon at $\delta_{c} 75.5(\mathrm{C}-20)$, one methine $s p^{2}$ at $\delta_{c} 124.8(\mathrm{C}-24)$ and one quartenary $s p^{2}$ carbon at $\delta_{c}$ 131.7 (C-25). The HMBC cross-peaks (Fig. 2) from $\mathrm{CH}_{3}-$ $28\left(\delta_{\mathrm{H}} 0.91\right), \mathrm{CH}_{3}-29\left(\delta_{\mathrm{H}} 0.81\right)$, and the methylene protons at $\mathrm{H}-2\left(\delta_{\mathrm{H}} 1.43\right)$ to the oxymethine carbon at C-3 ( $\delta_{c}$ 76.4) indicated the presence of a hydroxy group at $\mathrm{C}-3$. The correlation which was arising from $\mathrm{CH}_{3}-21$ $\left(\delta_{\mathrm{H}} 1.13\right)$ and $\mathrm{CH}_{3}-22\left(\delta_{\mathrm{H}} 1.44\right)$ to $\mathrm{C}-20$ ( $\left.\delta_{\mathrm{C}} 75.5\right)$ confirm that another hydroxy group at $\mathrm{C}-20$. The position of a double bond at C-24/C-25 evidenced by the correlation between $\mathrm{CH}_{3}-26\left(\delta_{\mathrm{H}} 1.66\right), \mathrm{CH}_{3}-27\left(\delta_{\mathrm{H}} 1.59\right)$, and $\mathrm{H}-23$ ( $\left.\delta_{H} 2.02\right)$ to $C-24$ ( $\left.\delta_{c} 124.8\right)$ and $C-25$ ( $\left.\delta_{c} 131.7\right)$. The conformation of $\mathrm{C}-3$ was assigned as $\beta$ based on coupling constant of $\mathrm{H}-3(\mathrm{~J}=4.5 \mathrm{~Hz})$ [33-34]. These functionalities accounted for one of five total degrees of unsaturation, and the remaining four degrees of unsaturation were consistent with the triterpenoid skeleton. A comparison of the NMR data of $\mathbf{4}$ with dammar-24-en-3 $\beta, 20$-diol $[30,35]$ revealed that the structures of the two compounds were very similar; consequently, compound $\mathbf{4}$ was identified as dammar24-en-3 $\beta, 20$-diol.

Compound $\mathbf{5}$ was obtained as a colorless oil. Its molecular composition $\mathrm{C}_{30} \mathrm{H}_{50} \mathrm{O}_{2}$, was established from the NMR data (Table 1). The ${ }^{1} \mathrm{H}-\mathrm{NMR}\left(\mathrm{CDCl}_{3} 600 \mathrm{MHz}\right)$ spectrum showed similar with 4 . The difference was no signal for oxymethin at $\delta_{\mathrm{H}} 3.37(1 \mathrm{H}, \mathrm{t}, J=4.5 \mathrm{~Hz}, \mathrm{H}-3)$, replace by carbonyl ketone. Indicate that oxidation product of $\mathbf{4}$ has formed.

The cytotoxic activity of the isolated compounds 14 and semi-synthetic compound 5 was evaluated against the P-388 murine leukemia cells according to a method described [23-24,28]. Artonin E (IC $50.75 \mu \mathrm{g} / \mathrm{mL})$ was used as positive control [27]. The results are shown in Table 2. Among all dammarane-type triterpenoid compounds, (E)-25-hydroperoxydammar-23-en-3 $\beta, 20$ diol (3), having a hydroperoxy group and straight side chain, showed the strongest activity among the dammarane-type triterpenoids tested, whereas 3-epi- cabraleahydroxy lactone (2) showed weak activity, indicated the releasing of three carbons and lactonization in side chain, significantly decreasing the cytotoxic activity. These results suggested that a hydroperoxy group in the side chain may be some important structural features for cytotoxic activity in dammarane-type triterpenoids.

\section{CONCLUSION}

Four dammarane-type triterpenoid compounds, dammar-24-en-3a-ol (1), 3-epi-cabraleahydroxy lactone (2), (E)-25-hydroperoxydammar-23-en-3 $\beta, 20$-diol (3), and dammar-24-en-3 $\beta, 20$-diol (4), were isolated from the methanolic extract of the stembark of Aglaia argentea. Compounds, 1-4, along with a known synthetic analog, 20-hydroxy-dammar-24-en-3-on (5), were evaluated their cytotoxic activity against P-388 murine leukemia cells in vitro. Among the dammaranetype triterpenoids, compounds 1, 3, 4 and 5 having opened side chain showed the stronger activity, where's compound $\mathbf{2}$ with cyclic side chain showed weak or no activity. In addition, compound $\mathbf{3}$ showed the strongest activity, indicate that hydroperoxy group at side chain increase cytotoxic activity.

\section{ACKNOWLEDGEMENT}

This investigation was financially supported by Directorate General of Higher Education, Ministry of Science, Technology and Higher Education, Indonesia (Postgraduate Grant, 2015-2016 by US). We thank Mrs. Suzany Dwi Elita at Department of Chemistry, Faculty of Mathemathics and Natural Sciences, Institute Technology Bandung, Indonesia for cytotoxicity bioassay.

\section{REFERENCES}

[1] Cao, J., Zhang, X., Qu, F., Guo, Z., and Zhao, Y., 2015, Dammarane triterpenoids for pharmaceutical use: A patent review (2005-2014), Expert Opin. Ther. Pat., 25 (7), 805-817.

[2] Kim, D.H., 2012, Chemical diversity of Panax ginseng, Panax quinquifolium, and Panax notoginseng, J. Ginseng Res., 36 (1), 1-15.

[3] Yang, W.Z., Hu, Y., Wu, W.Y., Ye, M., and Guo, D., 2014, Saponins in the genus Panax L. 
(Araliaceae): A systematic review of their chemical diversity, Phytochemistry, 106, 7-24.

[4] Connolly, J.D., and Hill, R.A., 2003, Triterpenoids, Nat. Prod. Rep., 20 (6), 640-659.

[5] Connolly, J.D., and Hill, R.A., 2008, Triterpenoids, Nat. Prod. Rep., 25 (4), 794-830.

[6] Kim, J.H., and Han, Y.N., 2011, Dammarane-type saponins from Gynostemma pentaphyllum, Phytochemistry, 72 (11-12), 1453-1459.

[7] Jia, L., Zhao, Y., and Liang, X.J., 2009, Current evaluation of the millennium phytomedicineginseng (II): Collected chemical entities, modern pharmacology, and clinical applications emanated from traditional Chinese medicine, Curr. Med. Chem., 16 (22), 2924-2942.

[8] Kuo, R.Y., Qian, K., Morris-Natschke, S.L., and Lee, K.H., 2009, Plant-derived triterpenoids and analogues as antitumor and anti-HIV agents, Nat. Prod. Rep., 26 (10), 1321-344.

[9] Man, S., Gao, W., Zhang, Y., Huang, L., and Liu, C., 2010, Chemical study and medical application of saponins as anti-cancer agents, Fitoterapia, 81 (7), 703-714.

[10] Pannell, C.M., 1992, A Taxonnomic Monograph of the Genus Aglaia Lour. (Meliaceae), Kew Bulletin Additional Series 16, London, H.M.S.O.

[11] Proksch, P., Edrada, R., Ebel, R.A., Bohnenstengel, F.I., and Nugroho, B.W., 2001, Chemistry and biological activity of rocaglamide derivatives and related compounds in Aglaia species (Meliaceae), Curr. Org. Chem., 5 (9), 923-938.

[12] Joycharat, N., Thammavong, S., Voravuthikunchai, S.P., Plodpai, P., Mitsuwan, W., Limsuwan, S., and Subhadhirasakul, S., 2010, Chemical constituents and antimicrobial properties of the essential oil and ethanol extract from the stem of Aglaia odorata Lour, Nat. Prod. Res., 28 (23), 2169-2172.

[13] Liu, S., Liu, S.B., Zuo, W.J., Guo, Z.K., Mei, W.L., and Dai, H.F., 2014, New sesquiterpenoids from Aglaia odorata var. microphyllina and their cytotoxic activity, Fitoterapia, 92, 93-99.

[14] Cai, X.H., Wang, Y.Y., Zhao, P.J., Li, Y., and Luo, X.D., 2010, Dolabellane diterpenoids from Aglaia odorata, Phytochemistry, 71 (8-9), 1020-1024.

[15] Yodsaoue, O., Sonprasit, J., Karalai, C., Ponglimanont, C., Tewtrakul, S., and Chantrapromma, S., 2012, Diterpenoids and triterpenoids with potential anti-inflammatory activity from the leaves of Aglaia odorata, Phytochemistry, 76, 83-91.

[16] Ishibashi, F., Satasook, C., Isman, M.B., and Towers, G.H.N., 1993, Insecticidal $1 \mathrm{H}$ cyclopentatetrahydro[b]benzofurans from Aglaia odorata, Phytochemistry, 32 (2), 307-310.
[17] Wu, T.S., Liou, M.J., Kuoh, C.S., Teng, C.M., Nagao, T., and Lee, A.H., 1997, Cytotoxic and antiplatelet aggregation principles from Aglaia elliptifolia, J. Nat. Prod., 60 (6), 606-608.

[18] Nugroho, B.W., Edrada, R.A., Wray, V., Witte L., Bringmann, G., Gehling, M., and Proksch, P., 1999, An insecticidal rocaglamide derivatives and related compounds from Aglaia odorata (Meliaceae), Phytochemistry, 51 (3), 367-376.

[19] Wang, B.G., Ebel, R., Wang, C.Y., Edrada, R.A., Wray, V., and Proksch, P., 2004, Aglacins I-K, three highly methoxylated lignans from Aglaia cordata, J. Nat. Prod., 67 (4), 682-684.

[20] Sianturi, J., Harneti, D., Darwati, Mayanti, T., Supratman, U., and Awang, K., 2016, A New (-)5',6-dimethoxyisolariciresinol-(3",4"-dimethoxy)-3aO- $\beta$-D-glucopyranoside from the bark of Aglaia eximia (Meliaceae), J. Nat. Prod. Res., 30 (19), 2204-2208.

[21] Roux, D., Martin, M., Adeline, M., Sevenet, T., Hadi, A.H., and Pais, M., 1998, Foveolins A and B, dammarane triterpenes from Aglaia foveolata, Phytochemistry, 49 (6), 1745-1748.

[22] Mohamad, K., Sévenet, T., Dumontet, V., Païs, M., Tri, M.V., Hadi, H., Awang, K., and Martin, M.T., 1999, Dammarane triterpenes and pregnane steroids from Aglaia lawii and A. tomentosa, Phytochemistry, 51 (8), 1031-1037.

[23] Harneti, D., Tjokronegoro, R., Safari, A., Supratman, U., Loong, X.M., Mukhtar, M.R., Mohamad, K., Awang, K., and Hayashi, H., 2012, Cytotoxic triterpenoids from the bark of Aglaia smithii (Meliaceae), Phytochem. Lett., 5 (3), 496499.

[24] Harneti, D., Supriadin, A., Ulfah, M., Safari, A., Supratman, U., Awang, K., and Hayashi, H., 2014, Cytotoxic constituents from the bark of Aglaia eximia (Meliaceae), Phytochem. Lett., 8, 28-31.

[25] Sianturi, J., Purnamasari, M., Darwati., Harneti, D., Mayanti, T., Supratman, U., Awang, K., and Hayashi, H., 2015, New bisamide compounds from the bark of Aglaia eximia (Meliaceae), Phytochem. Lett., 13, 297-301.

[26] Alley, M.C., Scudiero, D.A., Monks, A., Hursey, M.L., Czerwinski, M.J., Fine, D.L., Abbott, B.J., Mayo, J.G., Shoemaker, R.H., and Boyd, M.R., 1988, Feasibility of drug screening with panels of human tumor cell lines using a microculture tetrazolium assay, Cancer Res., 48 (3), 589-601.

[27] Hakim, E.H., Achmad, S.A., Juliawaty, L.D., Makmur, L., Syah, Y.M., Aimi, A., Kitajima, M., Takayama, H., and Ghisalberti, E.L., 2007, Prenylated flavonoids and related compounds of the Indonesian Artocarpus (Moraceae), J. Nat. Med., 61 (2), 229-236. 
[28] Sahidin, Hakim, E.H., Juliawaty, L.D., Syah, Y.M., Din, L.B., Ghisalberti, E.L., Latip, J., Said, I.M., and Achmad, S.A., 2005, Cytotoxic Properties of Oligostilbenoids from the Tree Barks of Hopea dryobalanoides, Z. Naturforsch., C: Biosci., 60 (910), 723-727.

[29] Xie, B.J., Yang, S.P, Chen, H.D., and Yue, J.M., 2007, Agladupols A-E, triterpenoids from Aglaia duperreana, J. Nat. Prod., 70 (9), 1532-1535.

[30] Zhang, F., Wang, J.S., Gu, Y.C., and Kong, L.Y., 2010, Triterpenoids from Aglaia abbreviata and their cytotoxic activities, J. Nat. Prod., 73 (12), 20422046.

[31] Esimone, C.O., Eck, G., Nworu, C.S., Hoffmann, D., Überla, K., and Proksch, P., 2010, Dammarenolic acid, a secodammarane triterpenoid from Aglaia sp. shows potent anti-retroviral activity in vitro, Phytomedicine, 17 (7), 540-547.
[32] Awang, K., Loong, X.M., Leong, K.H., Supratman, U., Litaudon, M., Mukhtar, M.R., and Mohamad, K., 2012, Triterpenes and steroids from the leaves of Aglaia exima (Meliaceae), Fitoterapia, 83 (8), 1391-1395.

[33] Breitmaier, E., 2002, Structure Elucidation by NMR in Organic Chemistry: A Practical Guide, John Wiley and Sons, London, 1-249.

[34] Cysne, J.B., Braz-Filho, R., Assunção, M.V., Uchoa, D.E., Silveira, E.R., and Pessoa, O.D., 2006, ${ }^{1} \mathrm{H}$ and ${ }^{13} \mathrm{C}$ NMR spectral assignments of four dammarane triterpenoids from carnauba wax, Magn. Reson. Chem., 44 (6), 641-643.

[35] Phongmaykin, J., Kumamoto, T., Ishikawa, T., Suttisri, R., and Saifah, E., 2008, A new sesquiterpene and other terpenoid constituents of Chisocheton penduliflorus, Arch. Pharmacal Res., 31 (1), 21-27. 